\title{
LEd-SpEC: SPECTROSCOPIC DETECTION OF WATER CONTAMINANTS USING GLOW DISCHARGES FROM LIQUID MICROELECTRODES
}

\author{
Chester G. Wilson and Yogesh B. Gianchandani \\ Department of Electrical and Computer Engineering, University of Wisconsin, Madison
}

\begin{abstract}
This paper reports on the detection of trace contaminants in water by spectroscopy of micro glow discharges that operate in air using liquid electrodes. A liquid electrode spectral emission chip (LEd-SpEC) has been developed to perform this function. Liquid from the cathode is sputtered into the discharge, and can be utilized for spectroscopic detection of impurities. Using a commercial spectrometer, measuring $\mathrm{Na}$ concentrations $<10 \mathrm{ppm}(\mathrm{mg} / \mathrm{l})$, and lead concentrations of $5 \mathrm{ppm}$. The relative intensity of certain spectral lines is shown to be a suitable measure of impurity concentration over several orders of magnitude. It is also shown that the addition of acids to lower the $\mathrm{pH}$ of the liquid solution increases the sensitivity of impurity detection. This device can also be used as a customizable optical source for UV and visible wavelengths.
\end{abstract}

\section{INTRODUCTION}

With threats to potable water sources from industrial and biochemical pollutants, diagnostic tools that can provide rapid on-site tests for contaminants are of significant interest. Presently, water quality assessment is a relatively elaborate process, requiring sample transportation and laboratory analysis at centralized locations. This analysis is typically done with a plasma spectrometer, a machine which varies in size from a table top model to room size. The water sample is sprayed into a high frequency RF inductively coupled plasma, and atomic transitions of the impurities are analyzed to determine the composition and quantity of the water impurities. This is similar to detection of gases in plasmas.

A variety of other methods are available for diagnosis of gas and water impurities, several of which have been miniaturized with MEMS technology. Mass spectrometers operate by measuring the ratio of ion mass to charge for gases, using a variety of techniques. Quadrupole mass spectrometers have an ion source, a quadrupole electrostatic lens to focus the ion flow, and an array of detectors. Ions with smaller mass to charge ratios are electrostatically deflected more. A micromachined quadrupole mass spectrometer was reported with 500 micron diameter quadrupole electrodes[1]. Time of flight mass spectrometers ionize gas atoms quickly, accelerate the ions, typically electrostatically, and measure their time of flight, which is a function of ion mass. Ion mobility mass spectrometers have the capability of operating at atmospheric pressure. These devices ionize gas using DC or RF voltage, or lasers.
Separation of species is based on their different mobilities in a background gas. An RF based ion mobility spectrometer has recently been miniaturized [2]. Gas chromatographs separate different gasses in a carrier gas flowing through a heated tube by exploiting differences in mobility for analysis. Detection mechanisms for chromatography vary, with gas ionization and spectral analysis being common. Recent efforts have been made to miniaturize these [3].

Microplasmas have been the focus of increasing research in recent years. We have reported DC microplasmas for silicon etching which were ignited between thin-film metal electrode features patterned on the substrate to be etched [4]. A DC microplasma generated in air at 2 Torr from two coplanar titanium electrodes is shown in Fig.1. The glow in these coplanar electrode microplasmas is confined to the region over the cathode. Other efforts have been directed at miniaturizing inductively coupled plasmas for gas spectroscopy [5], and to utilize DC microplasmas as an optical emission source for gas chromatography [6]. Atomic transitions of metallic impurities are typically best detected from spectroscopic analysis of DC plasma emissions [7]. Work has also been done employing the water sample as a cathode, with a metallic anode for spectroscopic use [8], [9], and detecting water impurities through spectral information. An effort to implement this system in a MEMS device has been reported very recently [10].

This paper reports on the detection of trace contaminants in water by spectroscopy of micro glow discharges that operate in air using two liquid electrodes (i.e., both the anode and cathode are liquid). A liquid electrode spectral emission chip (LEd-SpEC) has been developed to perform this function. Liquid from the cathode is sputtered into the discharge, and can be utilized for spectroscopic detection of impurities. This device can also be used as a customizable optical source for UV and visible wavelengths.

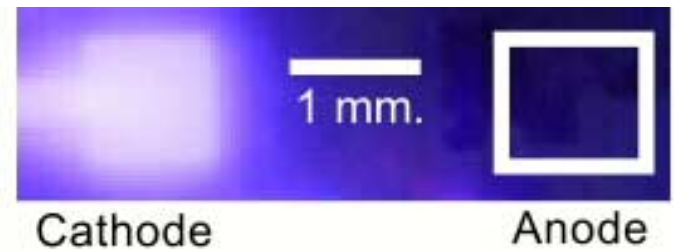

Fig. 1: Local confinement of a microplasma at 2 Torr and $410 \mathrm{~V}$ DC. 


\section{DEVICE STRUCTURE}

The LEd-SpEC uses the water sample, inserted into microchannels on a glass substrate, as the electrode material for generating a glow discharge for spectroscopic analysis (Fig. 2). This provides the following benefits: (i) the water sample and its impurities are effectively sputtered into the glow discharge, eliminating the need for spraying, which is otherwise necessary for detecting non-volatile contaminants; (ii) concern for electrode wear is eliminated since it is replenished by liquid flow; (iii) a continuous glow discharge can be sustained in air at atmospheric pressure, eliminating the need for a vacuum system. Thin film Pt features patterned within the channel provide lead transfers for the DC power supply. A $4.5 \mathrm{~mm}$ long channel covered by polyimide separates the exposed liquid in the $500 \mathrm{~m} \mathrm{x} 700$ $\mathrm{m}$ electrode regions from reservoirs, which do not participate in the glow discharge. The two liquid electrodes in each device are separated by $2.5 \mathrm{~mm}$. An optical fiber mounted above this region couples the device to a pagersized spectrometer (Ocean Optics USB-2000) connected to a data acquisition computer (Fig. 3). (The LEd-SpEC can accommodate a co-packaged micro spectrometer as well.)

\section{FABRICATION}

The device is fabricated on a glass substrate using a 4mask process (Fig. 4). The glass wafer is recessed by the first mask to define the sample confinement region. The second mask patterns thin film Pt electrodes, which perform lead transfer for the liquid electrodes. The third mask serves as a mold for a $30 \mu \mathrm{m}$ thick layer of electroplated $\mathrm{Cu}$ which defines the height of the flow channels. The last mask is used to pattern the layer of polyimide that covers the channels. Finally, the sacrificial $\mathrm{Cu}$ etched away, and a hydrophobic film applied to the inter-electrode surface to eliminate electrophoresis of water during prolonged operation [11].

\section{EXPERIMENTAL RESULTS}

Figure 5 shows the typical spectral output of a sample containing $5 \mathrm{ppm} \mathrm{Pb}$ dissolved in nitric acid with a $\mathrm{pH}$ of 3.2. A discharge current of $2.5 \mathrm{~mA}$ was used. Two dominant spectral lines characteristic to $\mathrm{Pb}$ are seen at 280 and at $405 \mathrm{~nm}$. The large spike at $358 \mathrm{~nm}$ is characteristic of $\mathrm{N}_{2}$. The lead spectra are a result of the impurities sputtered from the liquid electrode, while the $\mathrm{N}_{2}$ spectra which are seen in all discharges in this effort, are due to the air ambient.

Spectra of saline at $10 \mathrm{ppm}$ to $5000 \mathrm{ppm}$ concentrations are shown in Fig. 6. These show the primary $\mathrm{Na}$ contaminant line at $589 \mathrm{~nm}$ line, as well as $\mathrm{N}_{2}$. The $\mathrm{NH}$ and $\mathrm{OH}$ ionic lines are also visible are a result of the sputtered water, and its reaction with the atmosphere. In Fig. 6a, the case of $5000 \mathrm{ppm} \mathrm{Na}$, the sodium spectral intensity is so strong the spectrometer is saturated at $\mathrm{Na}$ wavelength, and the signal is much stronger than the $\mathrm{N}_{2}$ lines. In Fig. $6 \mathrm{~b}$, the case of $10 \mathrm{ppm} \mathrm{Na}$, the $\mathrm{N}_{2}$ lines dominate the $\mathrm{Na}$ lines.

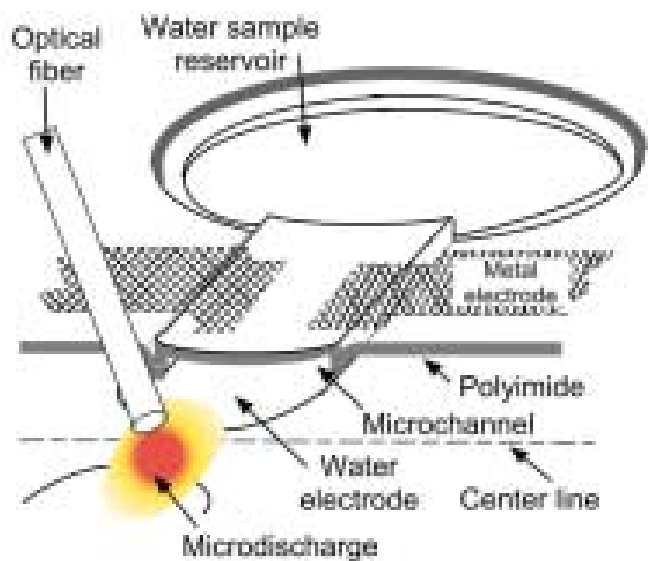

Fig. 2: Schematic of device concept.

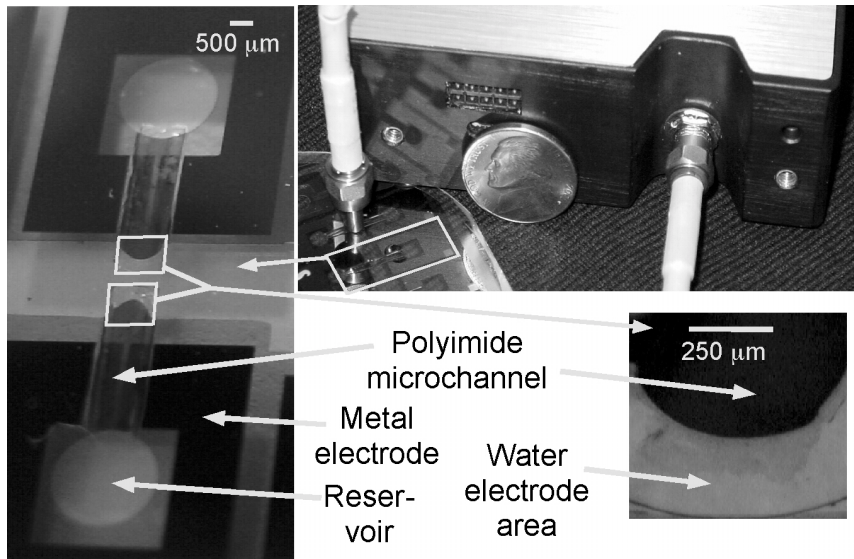

Fig. 3: Optical viewgraph of system and close-up view of the LEd-SpEC.

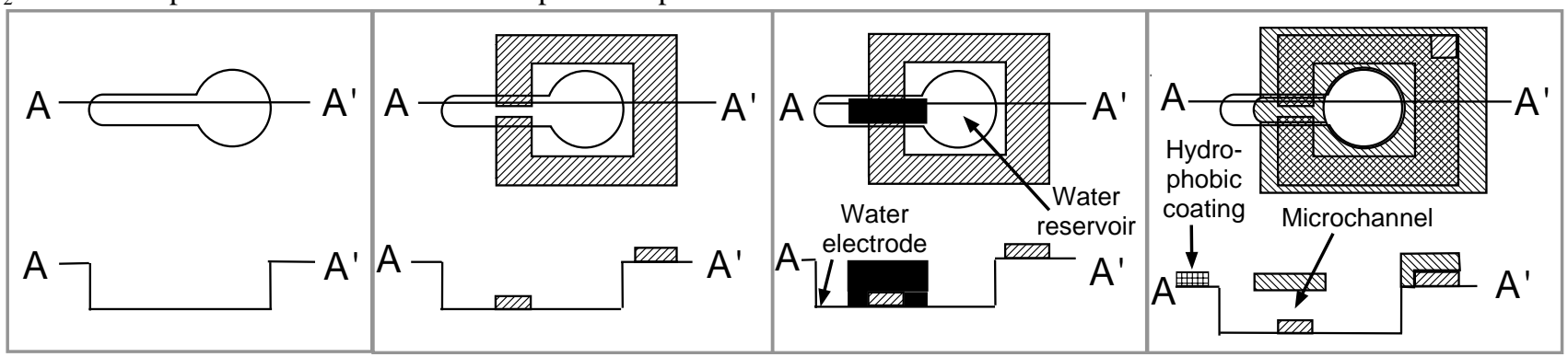

Fig. 4: Fabrication sequence: (i) recess glass substrate; (ii) pattern Pt lead transfer; (iii) electroplate Cu sacrificial layer for channel; (iv) pattern polyimide cover, etch sacrificial layer, apply hydrophobic film between electrodes. 


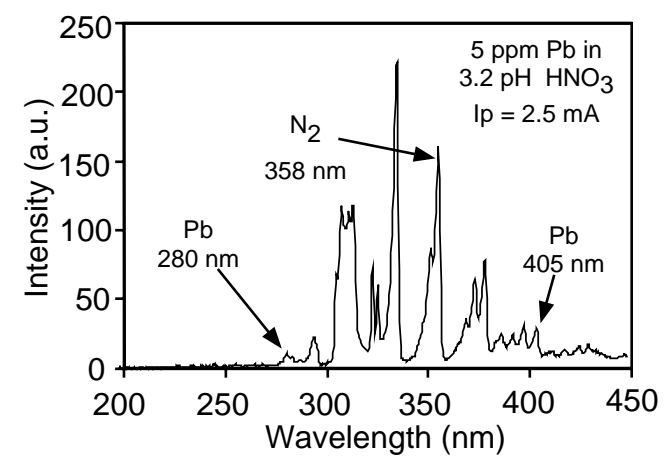

Fig. 5: Spectrum from sample containing $5 \mathrm{ppm} \mathrm{Pb.}$
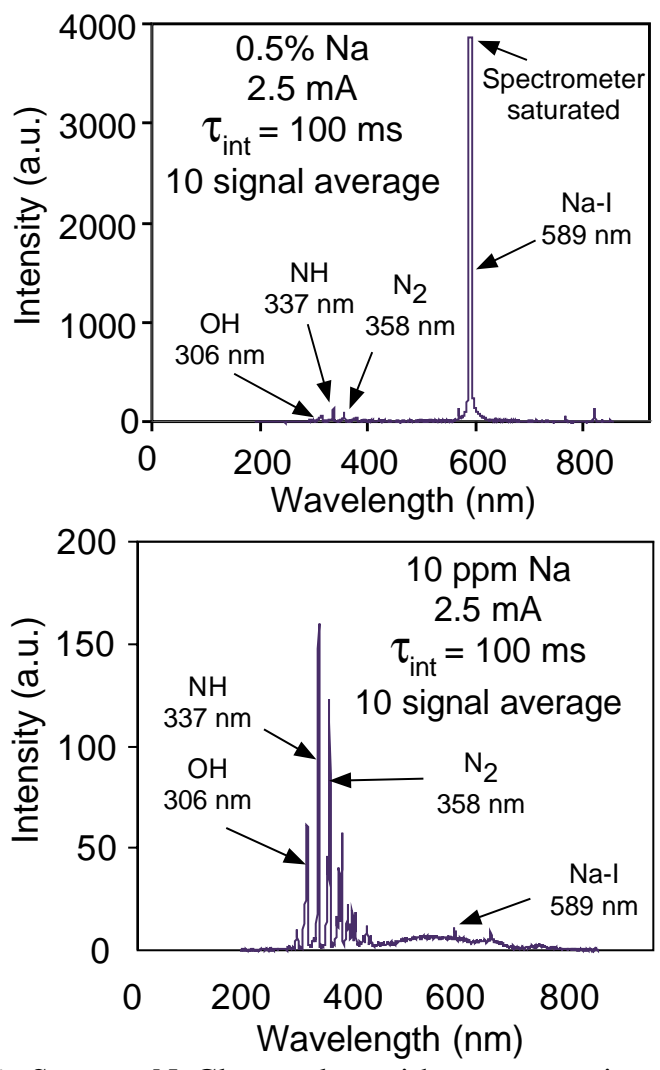

Fig. 6: Spectra $\mathrm{NaCl}$ samples with concentrations of (aupper) $5000 \mathrm{ppm}$, and (b-lower) $10 \mathrm{ppm}$.

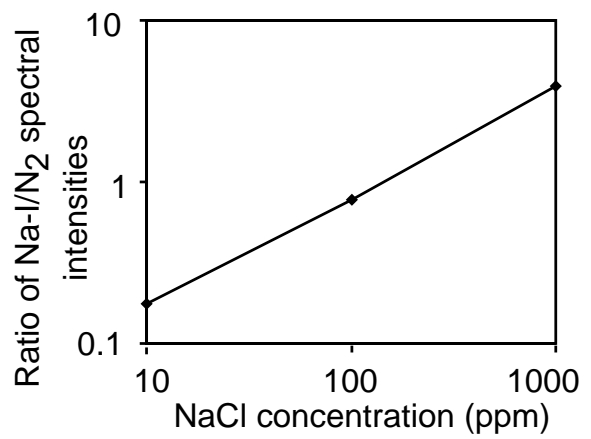

Fig. 7: Ratio of $\mathrm{Na}$ to $\mathrm{N}_{2}$ spectral intensities as a function of $\mathrm{NaCl}$ concentration.

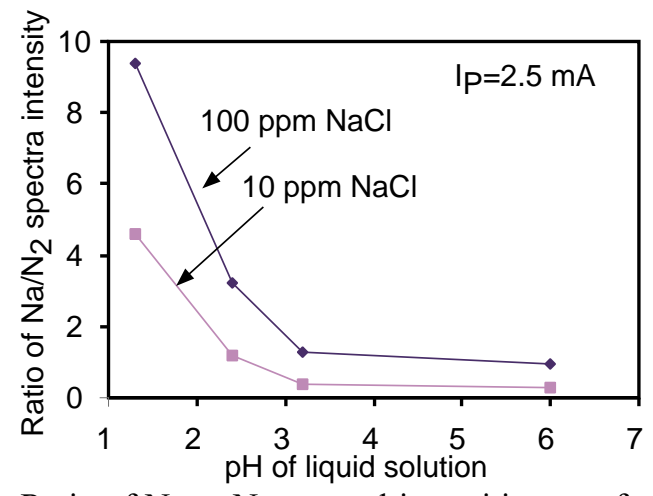

Fig. 8: Ratio of $\mathrm{Na}$ to $\mathrm{N}_{2}$ spectral intensities as a function of $\mathrm{pH}$ of the tested solution.

By measuring the ratio of the spectral intensities of the primary contaminant to $\mathrm{N}_{2}$, is it possible to determine the concentration of the impurity over a wide dynamic range. This is a valuable feature. Figure 7 shows the averaged ratio of $\mathrm{Na}$ to $\mathrm{N}_{2}$ spectra, for $\mathrm{Na}$ concentration ranging from 10 $\mathrm{ppm}$ to $1000 \mathrm{ppm}$. The ratio of the spectral intensities varies by almost two orders of magnitude.

The intensity of the sodium line can be increased by modifying the $\mathrm{pH}$ of the sample under test with nitric acid, while the intensity of the nitrogen line remains relatively unchanged. This results in the ratio of the $\mathrm{Na}$ to $\mathrm{N}_{2}$ spectral intensity lines to increase as the $\mathrm{pH}$ is decreased, which can be useful for extending the impurity detection limits (Fig 8).

To determine the source of the impurity ions in the glow discharge, the cathode of one device was filled with a 5000 ppm $\mathrm{NaCl}$, and the anode with DI water. This provided a spectrum similar to Fig. 6a, indicating that positive gas ions from the discharge sputtered the cathode. The spectrum obtained under the reverse polarity did not show the Na line (Fig. 9). This indicates that the dominant impurity delivery mechanism is sputtering from the cathode as opposed to fluid heating, and vaporization. This is an important feature of the LEd-SpEC because it permits inorganic impurities which are non-volatile to be introduced into the plasma. It eliminates the need for spraying the water into the plasma, which is the approach used in conventional devices.

The impact of varying the inter-electrode gap was tested using $5000 \mathrm{ppm} \mathrm{NaCl}$ on device variants (Fig. 10). Results showed a linear relationship between this dimension and the breakdown voltage. The voltage drop within the water channel can be substantial, but the lowering of the $\mathrm{pH}$ can reduce this. It is also possible to reduce the drive voltage by using a metal thin film electrode as the anode.

In addition to use in the detection of water impurities, the LEd-SpEC shows promise as a light source with a customizable spectrum. The device was tested with a 10,000 ppm chromium solution in 5\% nitric acid. Chrome provides multiple dominant spectral lines in the $200-207 \mathrm{~nm}$ range. It was found that the emission spectral power of the UV range increases as the device is put under vacuum. This is 
believed to be due to the increased electron energy in discharges at lower operating pressures. A device operated at 100 Torr, with $16 \mathrm{~mA}$ current and 650 volts was found to produce $8.3 \mathrm{~mW}$ of UV spectral power in the $195-235 \mathrm{~nm}$ wavelength range. This opens up some interesting possibilities for the development of on-chip UV sources.

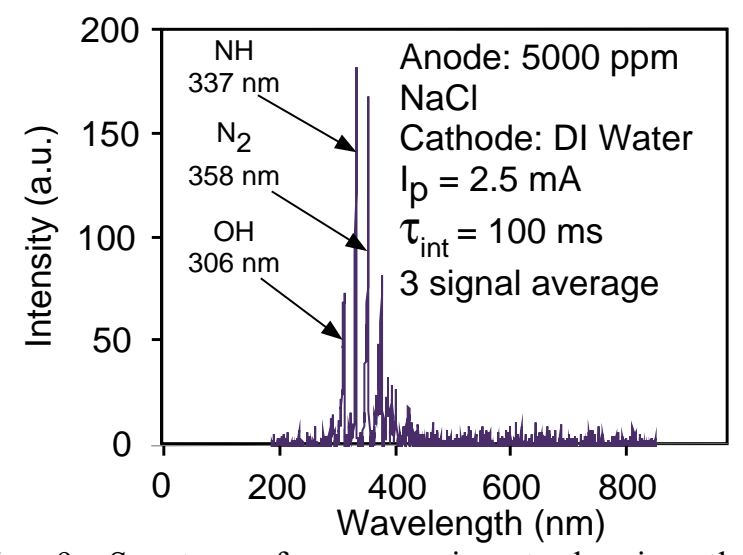

Fig. 9: Spectrum from experiment showing that all contamination measured is from cathode cell.

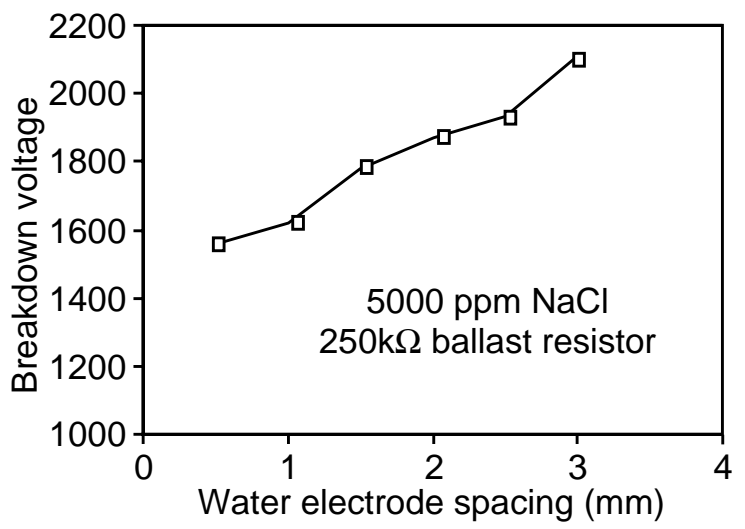

Fig. 10: Breakdown voltage dependence on inter-electrode gap for 5000 ppm NaCl.

\section{CONCLUSIONS}

The Led-SpEC device was shown to detect sodium impurities less than $10 \mathrm{ppm}$, and lead impurities at $5 \mathrm{ppm}$. The device operates by sputtering the cathode, which is the liquid sample under test. The relative concentration of sodium is a function of the ratio of the $\mathrm{Na} / \mathrm{N}_{2}$ spectral intensities, and varies over orders of magnitude. The addition of acid to lower the $\mathrm{pH}$ of the sample increases the intensity of the impurity line. The device shows promise as a possible on-chip UV source.

\section{ACKNOWLEDGEMENTS}

The authors would like to thank W. Zeltner for providing samples of $\mathrm{Pb}$-contaminated water.

\section{REFERENCES}

[1] S. Taylor, B. Srigengan, J.R. Gibson, D. Tindall, R. Syms, T.J. Tate, M.M. Ahmad, A Miniature Mass Spectrometer for Chemical and Biological Sensing, SPIE Proc. 4036, 2000, pp. 187-193

[2] R.A. Miller, E.G. Nazarov, G.A. Eiceman, A.T. King, "A MEMS Radio-Frequency Ion Mobility Spectrometer for Chemical Vapor Detection" Sensors and Actuators A, Vol. A91, no. 3, July 2001, pp. 301-12

[3] C.M. Yu, M. Lucas, J.C. Koo, P. Stratton, T, DeLima, E. Behymer, "A High Performance Hand-Held Gas Chromatograph", Micro-Electro-Mechanical Systems ASME International Mechanical Engineering Congress and Exposition, 1998, pp. 481-6

[4] C.G. Wilson, Y.B. Gianchandani, "Silicon micromachining using in-situ DC microplasmas" JMEMS 10(1) 3/01, pp. 50-54

[5] J.A. Hopwood, "A Microfabricated Inductively Coupled Plasma Generator”, JMEMS, 9(3), Sept. 2000, pp. 309-13

[6] J.C.T. Eijkel, H. Stoeri, A. Manz, "A dc Microplasma on a Chip Employed as an Optical Emission Detector for Gas Chromatography," Anal. Chem. 72 June 2000, pp. 2547-2552

[7] N.W. Routh, "DCP Advantages," Applied Research Laboratories Application Reports.

[8] T. Cserfalvi, P. Mezei, "Emission Studies on a Glow-Discharge in Atmospheric Air Using Water as a Cathode" J. Phys. D (Appl. Phys), 26 (1993) pp. 2184-2188

[9] R. K. Marcus, W.C. Davis, "An Atmospheric Pressure Glow Discharge Optical Emission Source for the Direct Sampling of Liquid Media," Anal. Chem., 73, (2001) pp. 2903-2910

[10] G. Jenkins, A. Manz, "Optical Emission Detection of Liquid Analytes Using a Micro-Machined D.C. Glow Discharge Device at Atmospheric pressure" $\mu$ TAS, Oct. 2001, pp. 349-350

[11] K. Handique, D.T. Burke, C.H. Mastrangelo, M.A. Burns, "Nanoliter-volume discrete drop injection and pumping in microfabricated chemical analysis systems" Proc. Solid State Sensors and Actuators Workshop, Hilton Head, '98, pp. 346-349 